\title{
Investigation of deformation of the cornea during tonometry using FEM
}

\author{
Bharathi R. B. ${ }^{1}$, Gopalakrishna Prabhu ${ }^{2}$, Ramesh S. Ve ${ }^{3}$, Rakshath Poojary ${ }^{4}$, S. Meenatchi Sundaram ${ }^{5}$ \\ ${ }^{1}$ Department of Electrical and Electronics Engineering, Manipal Institute of Technology, \\ Manipal Academy of Higher Education, India \\ ${ }^{2}$ Department of Electronics and Communication Engineering, Faculty of Engineering, \\ Manipal Academy of Higher Education, Manipal University Jaipur, India \\ ${ }^{3}$ Optometry Department, Manipal College of Health Professions, Manipal Academy of Higher Education, India \\ ${ }^{4}$ Department of Mechanical and Manufacturing Engineering, Manipal Institute of Technology, \\ Manipal Academy of Higher Education, India \\ ${ }^{5}$ Department of Instrumentation and Control Engineering, Manipal Institute of Technology, \\ Manipal Academy of Higher Education, India
}

\begin{tabular}{|c|c|}
\hline Article Info & ABSTRACT \\
\hline Article history: & A three-dimensional finite element model of the human eye is developed to \\
\hline Received Oct 1, 2019 & $\begin{array}{l}\text { evaluate the force which will be applied over the surface of cornea during } \\
\text { tonometry and gonioscopy tests. The standard tonometers and gonioscopy }\end{array}$ \\
\hline Revised Mar 26, 2020 & experiences deformation from $0.5 \mathrm{~mm}$ to $3 \mathrm{~mm}$ of the cornea is adopted \\
\hline Accepted May 8, 2020 & $\begin{array}{l}\text { during both point contact and boundary contact on the surface of the cornea. } \\
\text { The results demonstrate the maximum force experienced by the tonometer }\end{array}$ \\
\hline Keywords: & $\begin{array}{l}\text { with point contact at the center of the cornea for the maximum possible } \\
\text { deformation of the cornea during tonometry. The study also analyzes for }\end{array}$ \\
\hline $\begin{array}{l}\text { Finite element model } \\
\text { Gonioscopy }\end{array}$ & $\begin{array}{l}\text { the force experienced by the tonometer or goniolens with boundary layer } \\
\text { contact for the defined deformation of the cornea along the direction from } \\
\text { cornea towards the retina. }\end{array}$ \\
\hline
\end{tabular}

\section{Corresponding Author:}

S. Meenatchi Sundaram,

Department of Instrumentation and Control Engineering,

Manipal Institute of Technology, Manipal Academy of Higher Education,

Manipal, India.

Email: meena.sundar@manipal.edu

\section{INTRODUCTION}

Glaucoma refers to a group of diseases that have a common characteristic of optic neuropathy which in turn is associated with visual function failure. It causes characteristic progressive changes in the optic nerve head, visual field loss, or both. This ocular disease is also known as a silent thief of sight because there are no symptoms shown in the early stages. According to the World Health Organisation (WHO), glaucoma is known to be the second leading cause of blindness after cataracts. When the disease has reached the advanced or final stage it may lead to irreversible blindness. There is no cure for the disease but it can be halted or prolonged if it is diagnosed in the early stages. Tonometry is the important test procedure to determine the intraocular pressure (IOP) of the eye for the diagnosis of glaucoma. IOP is the only modifiable factor in glaucoma and the clinicians use this as an important clinical measure in the treatment of glaucoma [1]. Therefore, accurate, precise, constant measurement and monitoring of IOP is the cornerstone of glaucoma to preserve the patient's visual function. Similarly, the gonioscopy allows the examination of the anterior chamber angle and also helps detect the angle abnormalities that have diagnostic and therapeutic implications [2]. Hence the tonometry and gonioscopy tests are considered as one of the important test procedures to diagnose glaucoma. 
The present study involves the quantification of force on cornea exerted by the tonometer or goniolens during glaucoma screening test procedures. In an indentation or applanation tonometer and gonioscopy, the test will be conducted by deforming the cornea by applying the force to the outside of the globe. If the excess force is applied to the cornea, indentation or applanation tonometer displays a large amount of fluid during the measurement and may result in a rise in IOP measurement [3]. The gonioscopy may result in a change in the orientation of the iris relative to the cornea due to excessive force on cornea [4]. Hence the glaucoma test procedures should limit the force on the cornea so that the measurements are as accurate as possible with minimum or no damage.

Different types of applanation and indentation tonometers are existing in the market to measure the IOP of the eye. In applanation tonometers [3], when a flat surface is pressed against the cornea, the pressure in the eye may be measured by the force applied to the plane and the area of contact between the plane and the cornea. With the golden standard method Goldmann applanation tonometer, the force required to establish a standard area of contact of flattening of the inner corneal area of $7.35 \mathrm{~mm} 2$, which makes the diameter of the external area of corneal applanation to be $3.06 \mathrm{~mm}$, is measured.

Majzoub U [5] describes the amount of displacement of fluid in indentation tonometer is larger compared to the applanation tonometer despite considering the measures to reduce the amount of deformation of the cornea during the application of force on the cornea. Stocker [6] claims during the tonometry test when the weight of 22-26.5 gm is applied on the surface of the cornea, which may result in fluid being forced out of the anterior chamber angle at an increased rate. Hence the readings will be affected. Ishiguro et al., [7] claims that it will be difficult to continuously press the portion of cornea in Indentation gonioscopy to enlarge the angle portion. This method will also introduce much pain to the subject.

The human eye model is designed using the COMSOL Multiphysics simulation tool and the design is carried out referring to the journals [8-18] and based on the study authors have applied various boundary conditions and constraints on the model. Images are recorded by Corvis tonometer [19] enable to determine the biomechanical parameters of the eye. The amplitude of deformation, as well as the frequency of vibration of the eye, are also recorded. The study presents an algorithm for image analysis for the separation of eyeball reaction from the deformation of the cornea during the application of force on the cornea. Infrared light is an incident on the cornea and the reflected light is captured by the high-speed camera to analyze the deformation of the cornea during the measurement of IOP in air puff tonometer [20]. In schiotz tonometer [21], bending force on the plunger was analyzed against the indentation depth. The author concludes that force on the plunger and hence the resistance of the cornea to indentation decays with time and was also measured by the author. The author [22] Ooi et al., develops a boundary element model of human eye for analyzing the temperature changes during the treatment of laser thermokeratoplasty and is modelled using Beer-Lambert law. The intraocular pressure of eye is determined by Kempf et al., [23] in non-contact tonometry method by estimating the time required for the deformation of eye for a short air pulse. High speed camera is used to record the motion of the eye and step rise in displacement is obtained by assuming the nonlinear properties of human eye. The author Y. Kurita et al. [24] investigates the eye stiffness of different subjects with non invasive approach to understand the role of stiffness in the measurement of IOP during Goldmann applanation tonometry method. The author Friedman et al. [25] includes a system for finding the biomechanical properties of corneal tissue. It includes a light source and a microscopy system to scan the corneal tissue. The scattered light is filtered and hence three-dimensional profile indicates biomechanical properties of corneal tissue.

To measure IOP, a large series of both invasive and non-invasive tonometers are available in the market. The magnitude of deformation of the cornea during the tonometry test and gonioscopy depends upon the area of contact. The study of various tests and the literature [16-20] revealed that the amount of deformation of the cornea during any standard tonometry test will vary in the range from $0.1 \mathrm{~mm}$ to $3 \mathrm{~mm}$. In this work, simulation is carried out on the designed eye model using FEM based simulation software to quantify the force on cornea required to deform the cornea from $0.5 \mathrm{~mm}-3 \mathrm{~mm}$. The present study hence is aiming at the determination of force for the known prescribed deformation utilizing which new design of tonometer and gonioscopy may adapt the feature of indication during excess force on the cornea during tonometry and gonioscopy procedures.

\section{METHOD}

Part 2.1 describes the steps followed for the design of the human eye model. Part 2.2 expresses the force on the cornea for varying deformation of the cornea with point and boundary layer contact of the tonometer or goniolens. 


\subsection{Design of human eye model using FEM based software}

The human eye model is constructed using finite element-based method (FEM), comsol multiphysics simulation software. The designed model of the eye is isolated from the human head. The developed eye model mainly comprises of cornea, sclera, iris, lens, trabecular meshwork and ciliary body. The optic nerve is omitted from the model. The retina and the choroid are thin compared to that of the sclera and the thermal properties of choroid and retina are same as that of the sclera. Hence retina and choroid are modeled together with the sclera as one homogeneous region. Figure 1 lists the procedure followed during the design ofthe human eye model.

\subsubsection{Geometry}

Initially, an axisymmetric two-dimensional model of the human eye is constructed using COMSOL multiphysics simulation software. Figure 2 shows one half of the cross-section of the 2D human eye model in the XY plane. The geometric dimensions of the constructed eye model are as per standards [8]. To obtaina three-dimensional model, the 2D model of the eye is rotated by an angle of $360^{\circ}$ about the X-axis. In the COMSOL simulation tool, revolve operation is carried out in a $3 \mathrm{D}$ plane for $360^{\circ}$ to create $3 \mathrm{D}$ froma 2D model. The resulting 3D model view at $300^{\circ}$ with all interior structures are shown in Figure 3.

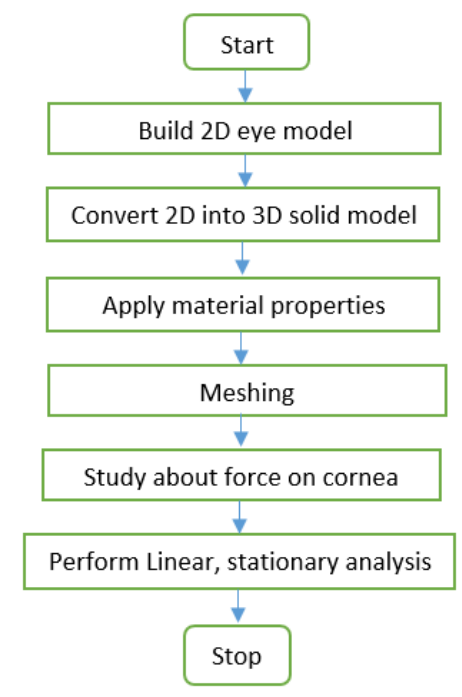

Figure 1. Steps followed for the design of the eye model

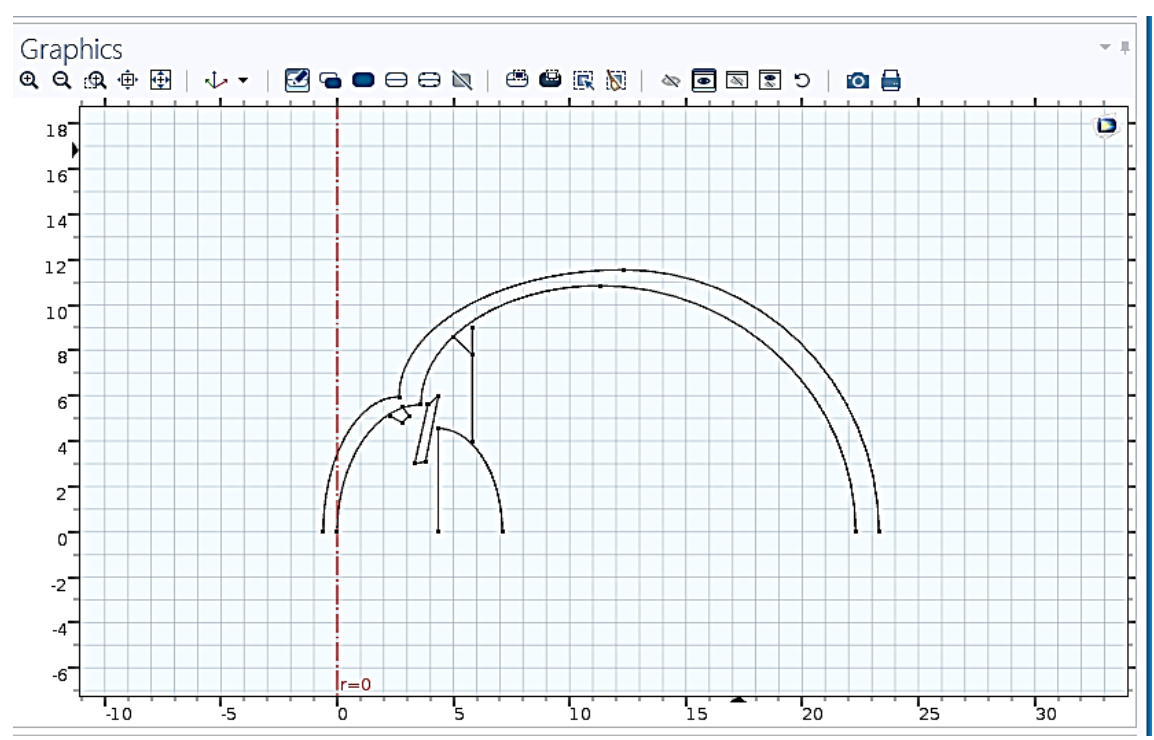

Figure 2. 2D model of eye 


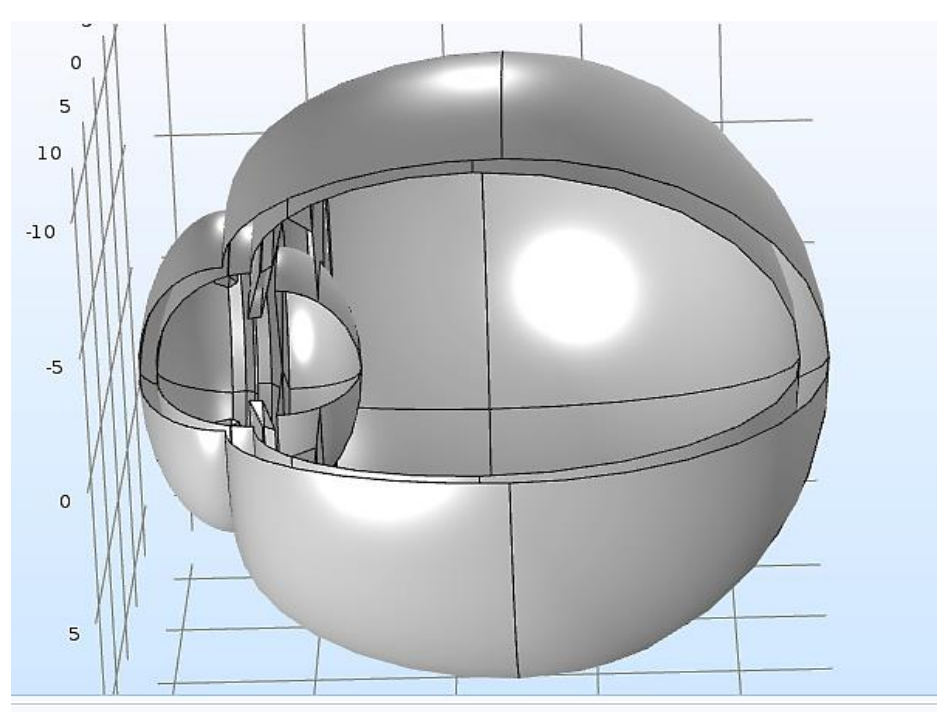

Figure 3. 3D model of eye at $300^{\circ}$

\subsubsection{Material properties}

Meanwhile, suitable material properties are applied to different parts of the eye model. The isotropic material properties of the complete eye model are detailed in Table 1. The properties of the human eye are as per standards [8].

Table 1. Material properties of the eye model

\begin{tabular}{|c|c|c|c|c|c|}
\hline Domain & $\begin{array}{c}\text { Thermal Conductivity } \\
\mathrm{K}(\mathrm{w} / \mathrm{m} / \mathrm{K})\end{array}$ & $\begin{array}{c}\text { Density } \rho \\
\left(\mathrm{Kg} / \mathrm{m}^{3}\right)\end{array}$ & $\begin{array}{l}\text { Heat Capacity at constant } \\
\text { Pressure }(\mathrm{J} / \mathrm{Kg} . \mathrm{K})\end{array}$ & Poissons ratio & $\begin{array}{l}\text { Young's modulus } \\
(\mathrm{Pa})\end{array}$ \\
\hline Cornea & 0.58 & 1050 & 4178 & 0.42 & $0.2 \mathrm{e} 6$ \\
\hline Iris, Sclera & 1.0042 & 1050 & 3180 & 0.4 & $0.9 \mathrm{e} 6$ \\
\hline Lens & 0.4 & 1000 & 3000 & & \\
\hline
\end{tabular}

\subsubsection{Boundary conditions}

During tonometry, the force on cornea results in the deformation of cornea. To analyze the behavior of cornea, three boundary conditions are applied to the developed model of the eye.

- Condition 1: The entire simulation is carried out with constant intraocular pressure of eye being 2933 $\mathrm{N} / \mathrm{m}^{2}$ (the equivalent of $22 \mathrm{mmHg}$ ) which is the normal IOP of the eye. Hence, the load defined at the internal surface of the eye as force per unit area is $2933 \mathrm{~N} / \mathrm{m}^{2}$ along X-direction.

- Condition 2: The posterior section of the eye, the sclera is fixed along $\mathrm{X}, \mathrm{Y}$ and $\mathrm{Z}$ directions and is specified in assumption 1.

$$
U=U x=0 ; V=U_{y}=0 ; W=U_{z}=0
$$

- Condition 3: The contact area of tonometer or goniolens with the eye is fixed. The deformation applied over the surface of the cornea along X-direction is varied and deformation of the cornea along $\mathrm{Y}$ and $\mathrm{Z}$ directions are zero as given in assumption 2.

$$
U=U x=\text { variable } \quad V=U_{y}=0 ; W=U_{z}=0
$$

\subsubsection{Mesh}

Complete eye geometry is divided into small elements called mesh and stationary analysis is carried over each individual mesh. The free tetrahedral mesh is fully automatic and can be applied over every geometry is utilized for meshing the eye model. While meshing, the minimum element size for the mesh is selected as $0.43 \mathrm{~mm}$, the maximum element size is $2.39 \mathrm{~mm}$, the maximum element growth rate is 1.5 and the curvature factor of the mesh is 0.6 . Finalized geometry has 7670 number of nodes, 42156 number of elements and average element quality is 0.6265 . Figure 4 describes the meshing of the eye model. 


\subsubsection{Solver}

The analysis is carried out with a Stationary linear solver with a relative tolerance of 0.0001 and minimum damping factor of 1e-4. The number of degrees of freedom solved for 183390. The output of the study is to evaluate the force required to provide prescribed displacement of the surface of the cornea along $\mathrm{X}, \mathrm{Y}$ and $\mathrm{Z}$ directions. The stress distribution within the eye for the prescribed displacement of point contact of tonometer along $\mathrm{X}$ direction is shown in Figure 5. The stress distribution will vary in the range of $5 \mathrm{MPa}-25 \mathrm{MPa}$ at the center of the cornea.

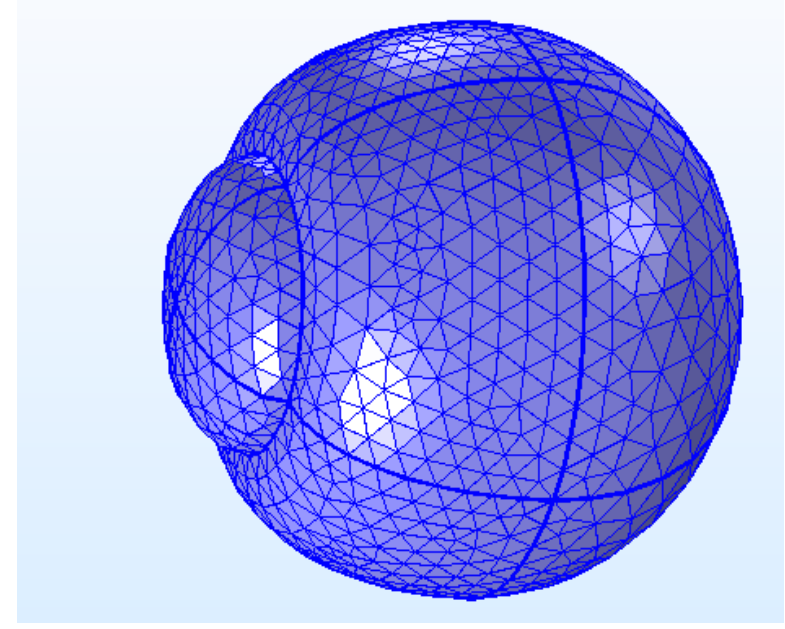

Figure 4. Tetrahedral mesh of eye

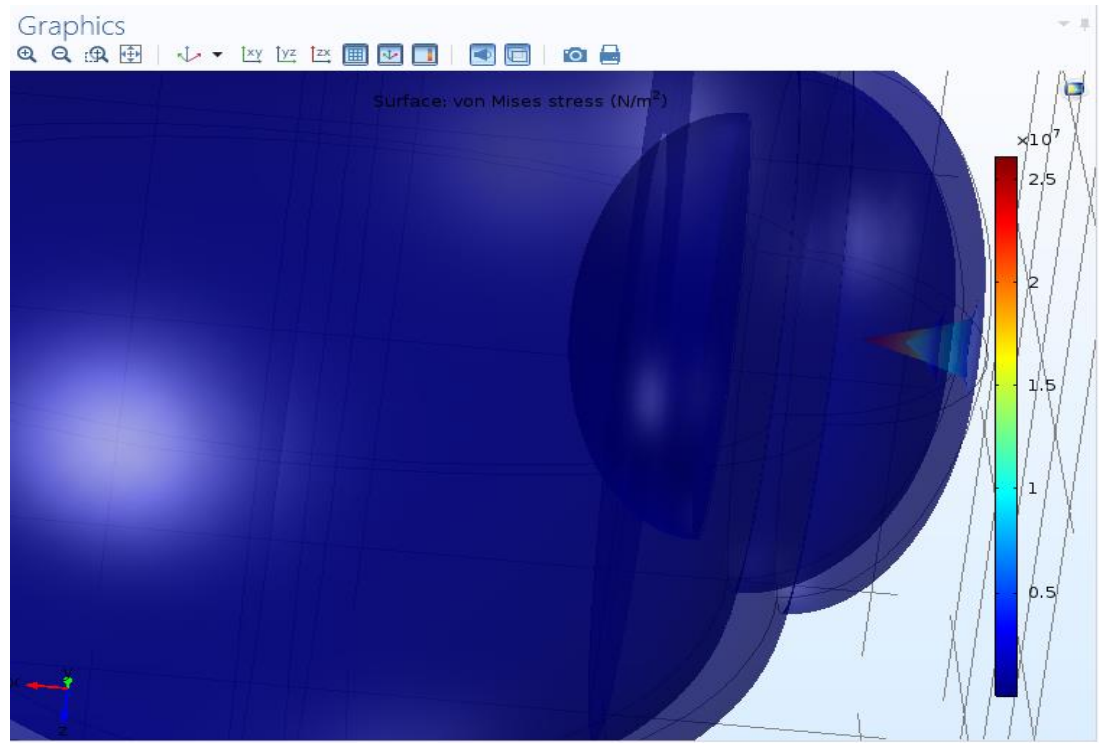

Figure 5. Stress distribution within eye

\subsection{Determination of force for the predetermined displacement of the cornea:}

Different types of tonometer and goniolens available in the market own different areas of contact on the cornea and hence claim with varying deformation of cornea. The study involves a point contact of tonometer and a standard size of $1 \mathrm{~mm}$ diameter boundary layer contact of tonometer or goniolens over the surface of the cornea. The simulation is carried out to find the influence of force upon the deformation of cornea. The deformation of the cornea during the application of force on the cornea using standard tonometer or goniolens vary from $0.5 \mathrm{~mm}$ to $3 \mathrm{~mm}$ [16-20]. Simulation is carried out with deformation of the cornea 
varying from $0.5 \mathrm{~mm}$ to $3 \mathrm{~mm}$ in steps of $0.5 \mathrm{~mm}$ as per standards. Thus, the maximum force on the cornea is explored in the simulation. The deformation of cornea not only depends on the amount of force applied but also upon the thickness of the cornea (CCT) and Intraocular Pressure (IOP) of the eye. The study is carried out at constant IOP of $22 \mathrm{mmHg}$ and the thickness of the cornea is considered to be $0.5 \mathrm{~mm}$ (for a normal eye).

\subsubsection{Force on the cornea for point contact of tonometer}

Here, the class of tonometer makes point contact with the surface of the cornea. The tonometer in the mode of point contact with the cornea is at the center of the cornea. The outer and the inner surface of cornea at the center are bounded by the prescribed displacement of $U_{x}=$ variable along $X$-direction. The force on the cornea for the prescribed displacement $(0.5 \mathrm{~mm}-3 \mathrm{~mm})$ for point contact of tonometer at the center of the cornea along $\mathrm{X}$-direction is investigated using linear stationary solver configuration.

\subsubsection{Force on the cornea for boundary layer contact of tonometer or goniolens}

The class of tonometer considered is in the form of a boundary layer of $0.02 \mathrm{~mm}$ thickness and a $0.5 \mathrm{~mm}$ radius spread uniformly over the center of the cornea. The material property assigned for the optic lens placed within the tonometer is a crown glass of a refractive index of 1.52 and is of low dispersion. The force on cornea responsible for the prescribed displacement $(0.5 \mathrm{~mm}-3 \mathrm{~mm})$ of the boundary layer contact of tonometer or goniolens along X-direction is analyzed using linear stationary solver configuration.

\section{RESULTS AND DISCUSSION:}

The applanation/indentation tonometer and gonioscopy results in the deformation of the cornea during the glaucoma test procedure. The innovation of new technology to measure eye pressure (tonometer) works on a variable area of applanation or variable force of the applanation principle. Any of these two technologies must not deform the cornea above the prescribed limit. Similarly, during the gonioscopy procedure, rotation of the goniolens must not disturb the normal functioning of the eye. The excessive force on the cornea may damage the internal parts of the eye. Hence new innovative technology to measure IOP (tonometer prototype) or gonioscopy should monitor the amount of force to deform the cornea. This study is focussed on different types of tonometers and goniolens which results in varying deformation of the cornea from $0.5 \mathrm{~mm}-3 \mathrm{~mm}$ and hence force required to provide the specified deformation of the cornea is evaluated. The area of contact between different types of tonometers is distinct. The simulation is carried out with two types of contacts of a tonometer, i.e. point contact of tonometer at the center of cornea and boundary layer contact of tonometer and goniolens with $0.02 \mathrm{~mm}$ thickness and $0.5 \mathrm{~mm}$ radius spread across the center of the cornea. The results are plotted using MATLAB curve fitting toolbox.

\subsection{Force on the cornea for point contact of tonometer}

The point contact of the tonometer at the center of the cornea with $1.5 \mathrm{~mm}$ deformation of cornea is indicated in Figure 6. The zoomed image illustrates the stress distribution at the point of contact of the tonometer over the surface of the cornea. Force $(\mathrm{N})$ along $\mathrm{X}, \mathrm{Y}, \mathrm{Z}$ direction against the displacement of the cornea $(\mathrm{mm})$ is display in the plot of Figure 7.

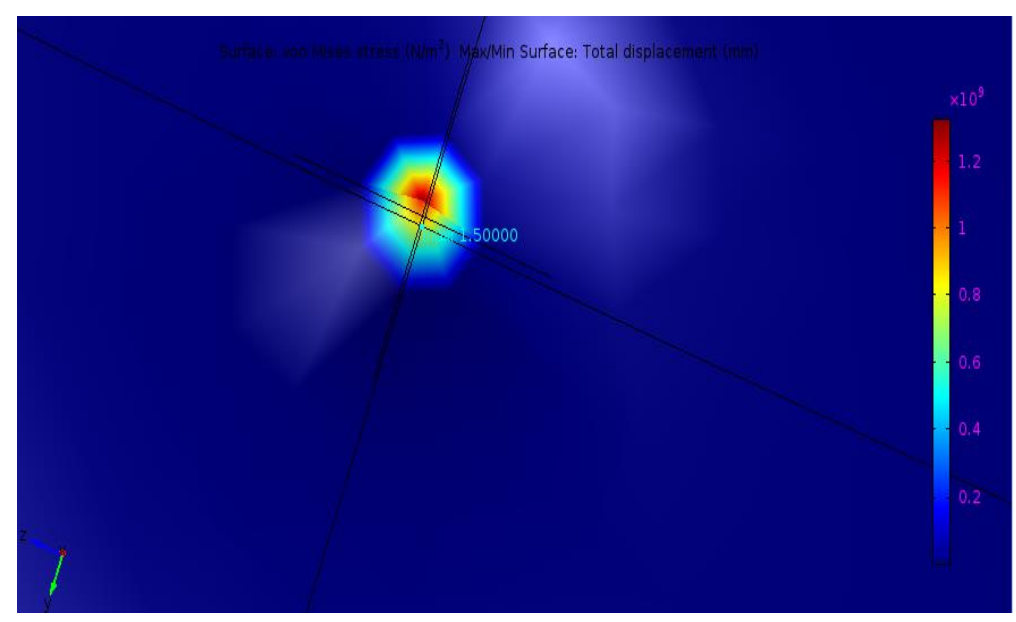

Figure 6. Point contact of tonometer with $1.5 \mathrm{~mm}$ deformation 


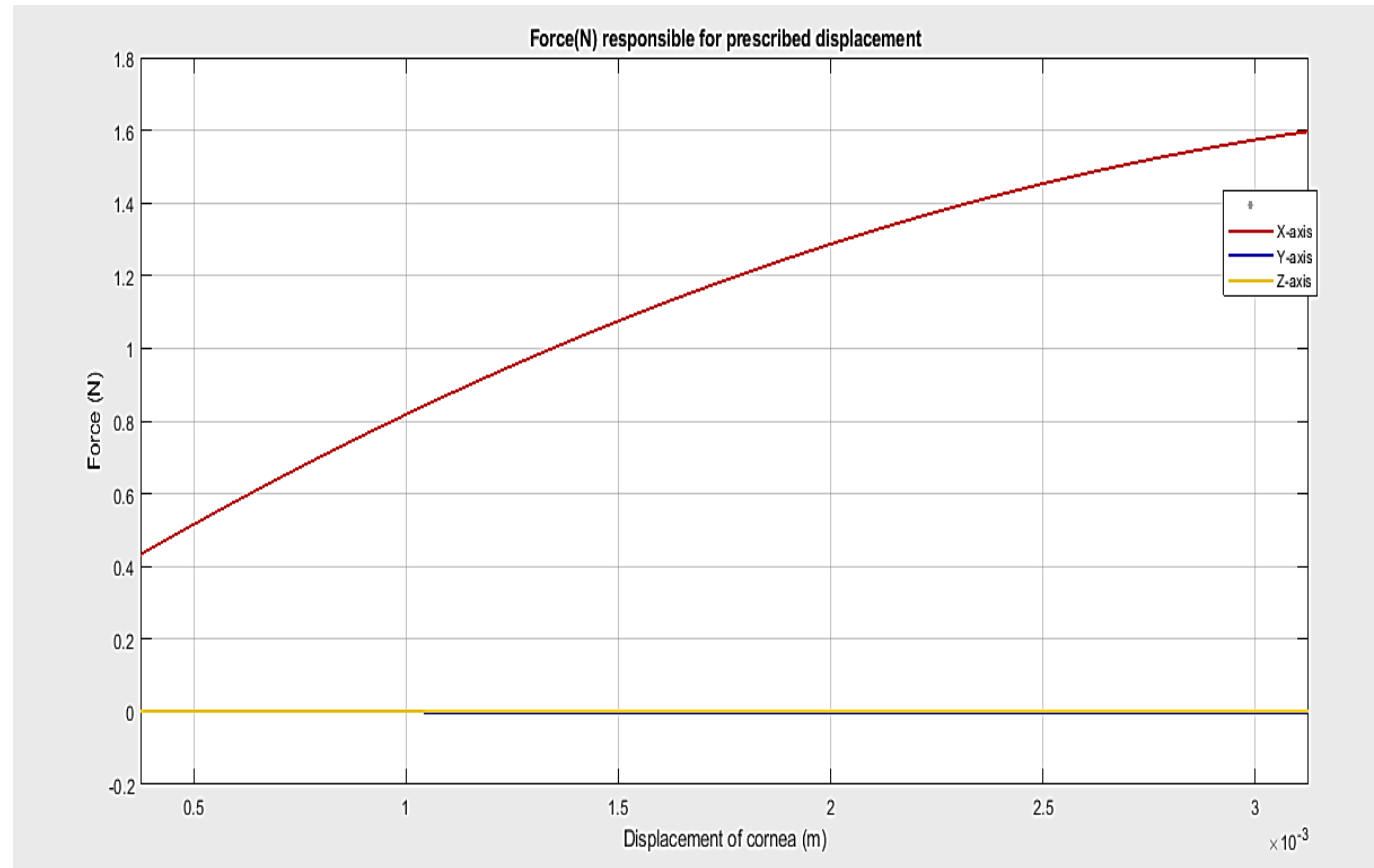

Figure 7. Force $(\mathrm{N})$ along $\mathrm{X}, \mathrm{Y}, \mathrm{Z}$ direction against the displacement of the cornea $(\mathrm{mm})$

The Figure 7 illuminates the force along $\mathrm{X}, \mathrm{Y}$ and $\mathrm{Z}$ direction when tonometer makes point contact with the cornea. The plot summarises that force F1 by the tonometer along $\mathrm{X}$-axis exponentially increases with an incremental increase in the displacement of the cornea and there is a negligible amount of force along $\mathrm{Y}$ and $\mathrm{Z}$ axes during displacement of the cornea along $\mathrm{X}$-axis. The plot concludes that a force of $0.5 \mathrm{~N}$ is experienced for $0.5 \mathrm{~mm}$ deformation of the cornea and a force of $1.6 \mathrm{~N}$ is experienced for $3 \mathrm{~mm}$ deformation of the cornea along the $\mathrm{X}$-axis with point contact of the tonometer. Hence, the force on cornea along the $\mathrm{X}$-axis increases with an increase in the deformation of cornea. The second-order polynomial equation for the force $\mathrm{F} 1$ along $\mathrm{X}, \mathrm{Y}$ and $\mathrm{Z}$ direction for point contact of tonometer at the center of the cornea is explained in (1).

$$
F 1=p 1 * x^{2}+p 2 * x+p 3
$$

where coefficients for $(1)$ are $\mathrm{p} 1=-0.07948, \mathrm{p} 2=0.396, \mathrm{p} 3=1.187$ and $\mathrm{x}$ is the displacement of cornea $(\mathrm{mm})$. In (1) can be justified since the value of $\mathrm{R}^{2}$ is 0.9858 which is close to unity.

\subsection{Force on the cornea for boundary layer contact of tonometer or goniolens}

The zoomed image shown in Figure 8, picturizes the deformation of the cornea by $0.5 \mathrm{~mm}$ due to the application of force over a boundary layer contact of tonometer along the X-direction. Figure 9 indicates the center and the 4 corner points of the boundary layer contact of the tonometer or goniolens. The force on cornea along $\mathrm{X}, \mathrm{Y}$ and $\mathrm{Z}$ directions $(\mathrm{F} 2, \mathrm{~F} 3, \mathrm{~F} 4)$ at the center and 4 corner points of boundary layer contacts of the tonometer is demonstrated in plots of Figure 10-12.

Figure 10 explains that force F2 along the $\mathrm{X}$-axis at the 4 corners of the boundary layer increases linearly and at the center decreases linearly in the negative direction with the displacement of the cornea. Hence, when the tonometer or goniolens is in the form of a boundary layer,force at the corners increases, while only at the centre decreases linearly. Figure 10 concludes that force on cornea F2 of up to $0.03 \mathrm{~N}$ is experienced at the 4 corners of the tonometer for $3 \mathrm{~mm}$ deformation of the cornea along the $\mathrm{X}$-axis. While the force F2 at the center of tonometer or goniolens during the measurement is very small and is close to zero. The first order linear polynomial equation for the force on cornea F2 is given in (2). The value of $R^{2}$ is used as an indicator to validate the model and it is accurate since $\mathrm{R}^{2}$ is unity and hence the defined equation for the force on the cornea can be validated. The Table 2 lists the coefficients $\mathrm{p} 1, \mathrm{p} 2$ for the force along $\mathrm{X}$-axis at the centre and at the four corners of the boundary contact of cornea.

$$
F 2=p 1 * x+p 2
$$




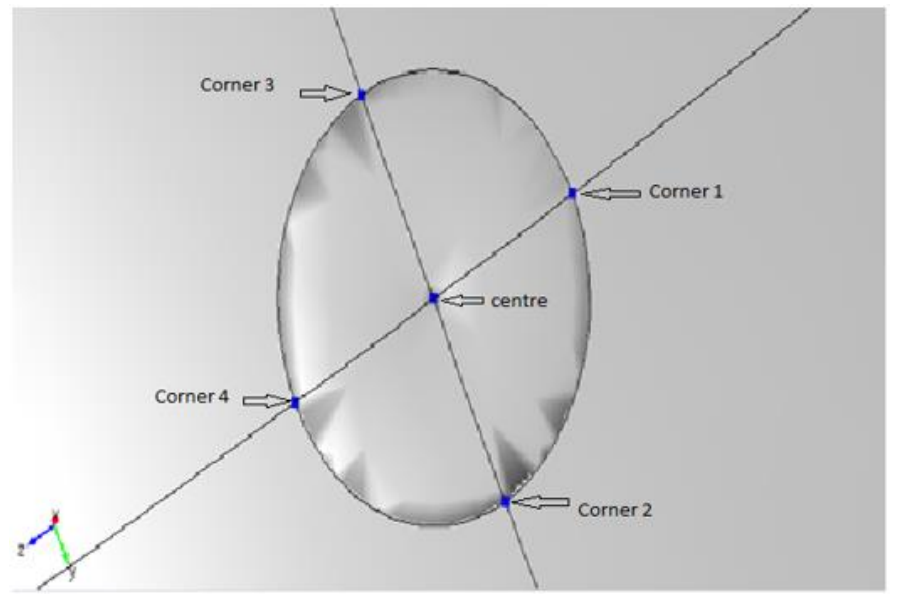

Figure 8 . Boundary layer contact of tonometer or goniolens with $0.5 \mathrm{~mm}$ deformation of cornea

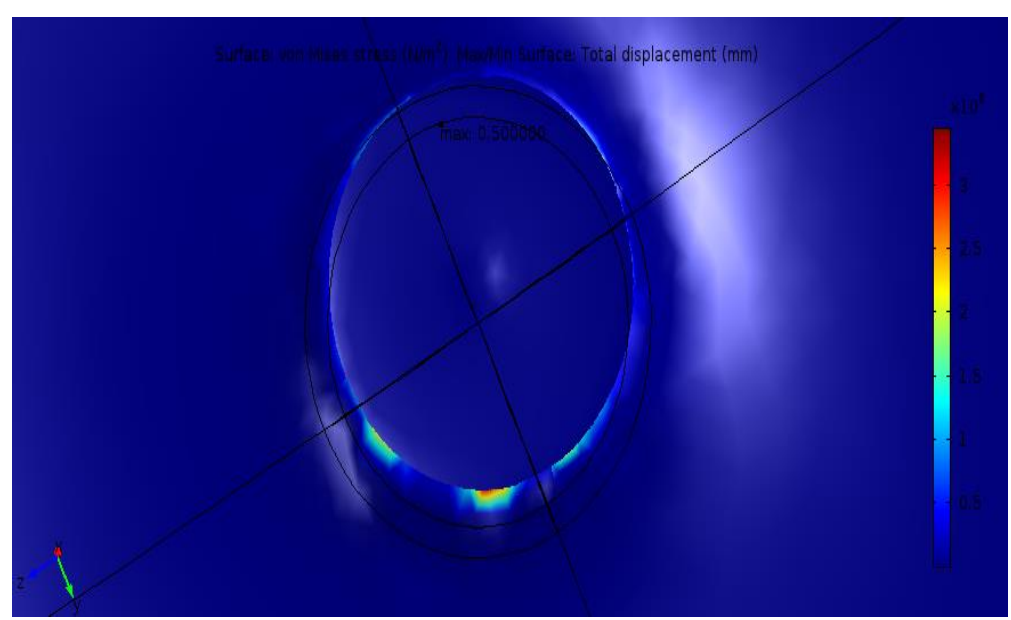

Figure 9. Representation of the boundary layer contact points 1 to 5 .

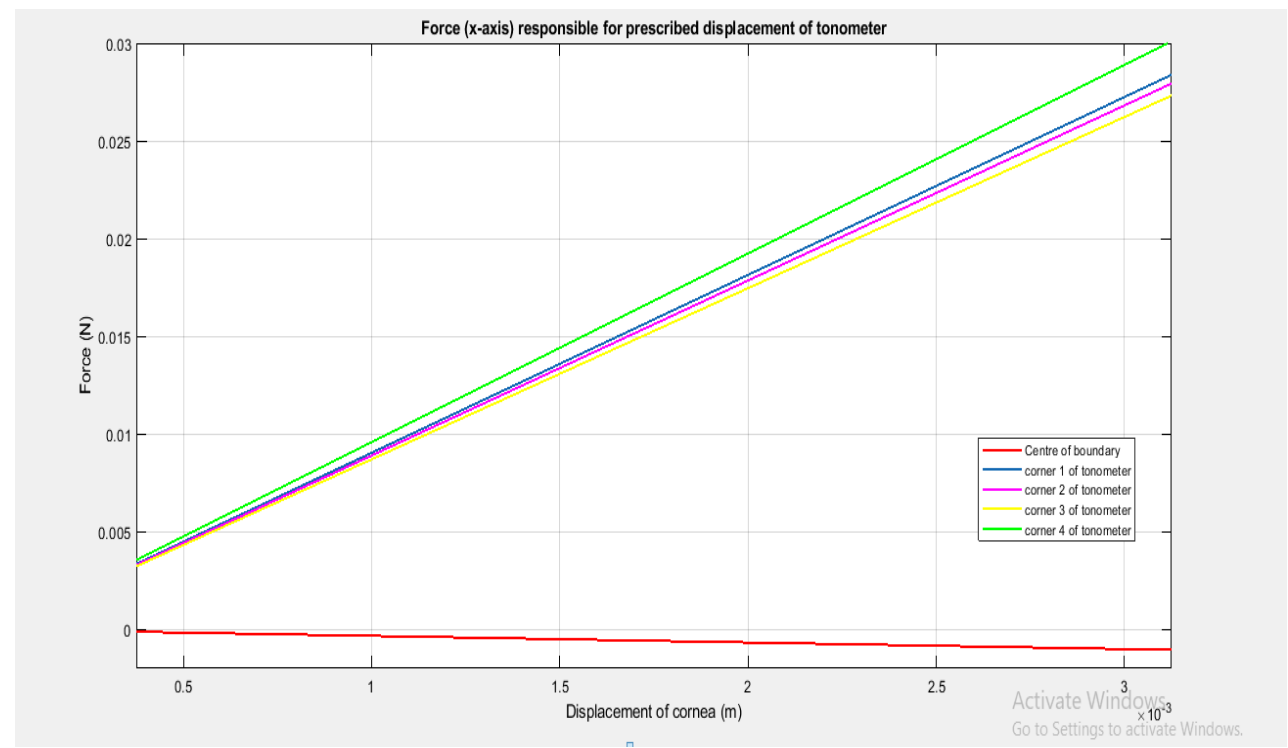

Figure 10. Force on the cornea $(\mathrm{N})$ at the center and 4 corner points of boundary layer along $\mathrm{X}$-axis 
Table 2. Coefficients (p1, p2) of (2) at the center and boundary points

\begin{tabular}{cccc}
\hline Boundary selection point & P1 & P2 & $R^{2}$ \\
\hline Centre & -0.0003154 & -0.00059 & 0.9994 \\
Corner 1 & 0.008517 & 0.01586 & 1 \\
Corner 1 & 0.008383 & 0.01561 & 1 \\
Corner 3 & 0.008198 & 0.01527 & 1 \\
Corner 4 & 0.009031 & 0.01682 & 1 \\
\hline
\end{tabular}

The Figure 11 indicates that force F3 along the Y-axis at corner 1 of the boundary layer is negative and hence acts in a direction opposite to $\mathrm{Y}$-axis and is linear concerning displacement of the cornea. The force F3 at the center and corner 2 of the boundary layer is negligible. The force F3 at corner 3 and corner 4 of the boundary layer increase linearly with the displacement of the cornea along the $\mathrm{X}$-axis. Plot explains that force on cornea F3 during tonometry and gonioscopy at the corners and center along Y-axis is negligible and is of the order of $\mathrm{mN}$. The first order linear polynomial equation for the force at the center and 4 corner points is indicated in (3). The model is authentic since the value of $\mathrm{R}^{2}$ is unity. The Table 3 lists the coefficients $\mathrm{p} 1, \mathrm{p} 2$ for the force along $\mathrm{Y}$-axis at the centre and at the four corners of the boundary contact of cornea.

$$
F 3=p 1 * x+p 2
$$

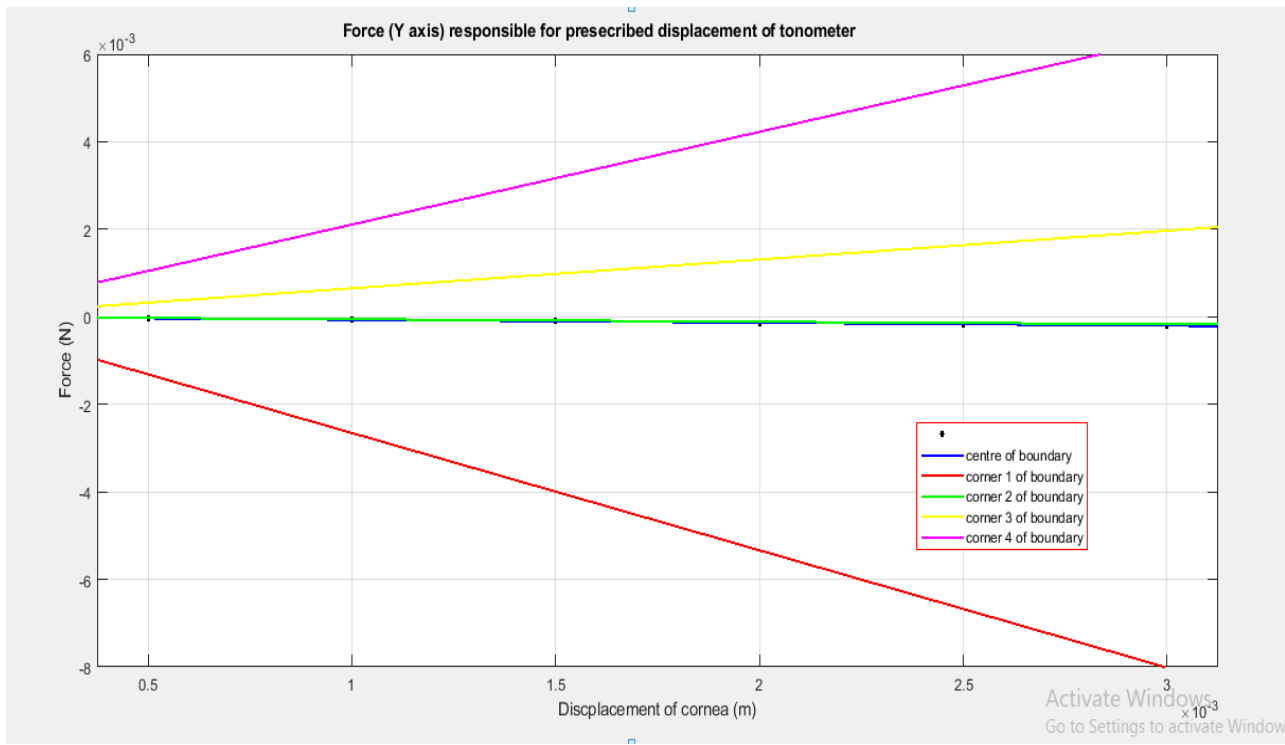

Figure 11. Force $(\mathrm{N})$ at the center and 4 corner points of boundary layer along $\mathrm{Y}$-axis

Table 3. Coefficients (p1, p2) of (3) at the center and boundary points

\begin{tabular}{cccc}
\hline Boundary selection point & P1 & P2 & $R^{2}$ \\
\hline Centre & $-6.286 \mathrm{e}-05$ & -0.0001173 & 0.9997 \\
Corner 1 & -0.002508 & -0.004666 & 1 \\
Corner 2 & $-5.282 \mathrm{e}-05$ & $-9.765 \mathrm{e}-05$ & 1 \\
Corner 3 & 0.0006153 & 0.001146 & 1 \\
Corner 4 & 0.001982 & 0.003697 & 1 \\
\hline
\end{tabular}

The Figure 12 signifies that the amount of force F4 along the Z-axis is almost zero at the center and at corner 1, corner 2 and corner 5 of the boundary layer force F4 is negative, acts in a direction opposite to $\mathrm{Z}$-axis and is linear with respect to displacement of the cornea. The force F4 at corner 3 is not linear concerning the displacement of the cornea. Figure 12 summarises that the force F4 acting at the corners and center of tonometer or goniolens is negligibly small and is of the order of $\mathrm{mN}$. The fourth-order linear polynomial equation for the force on cornea F4 along Z-axis as shown in (4). The value of $\mathrm{R}^{2}$ is unity. The table 4 lists the coefficients p1, p2, p3, p4 and p5 for the force along Z-axis at the centre and at the four corners of the boundary contact of cornea. 
$F 4=p 1 * x^{4}+p 2 * x^{3}+p 3 * x^{2}+p 4 * x+p 5$

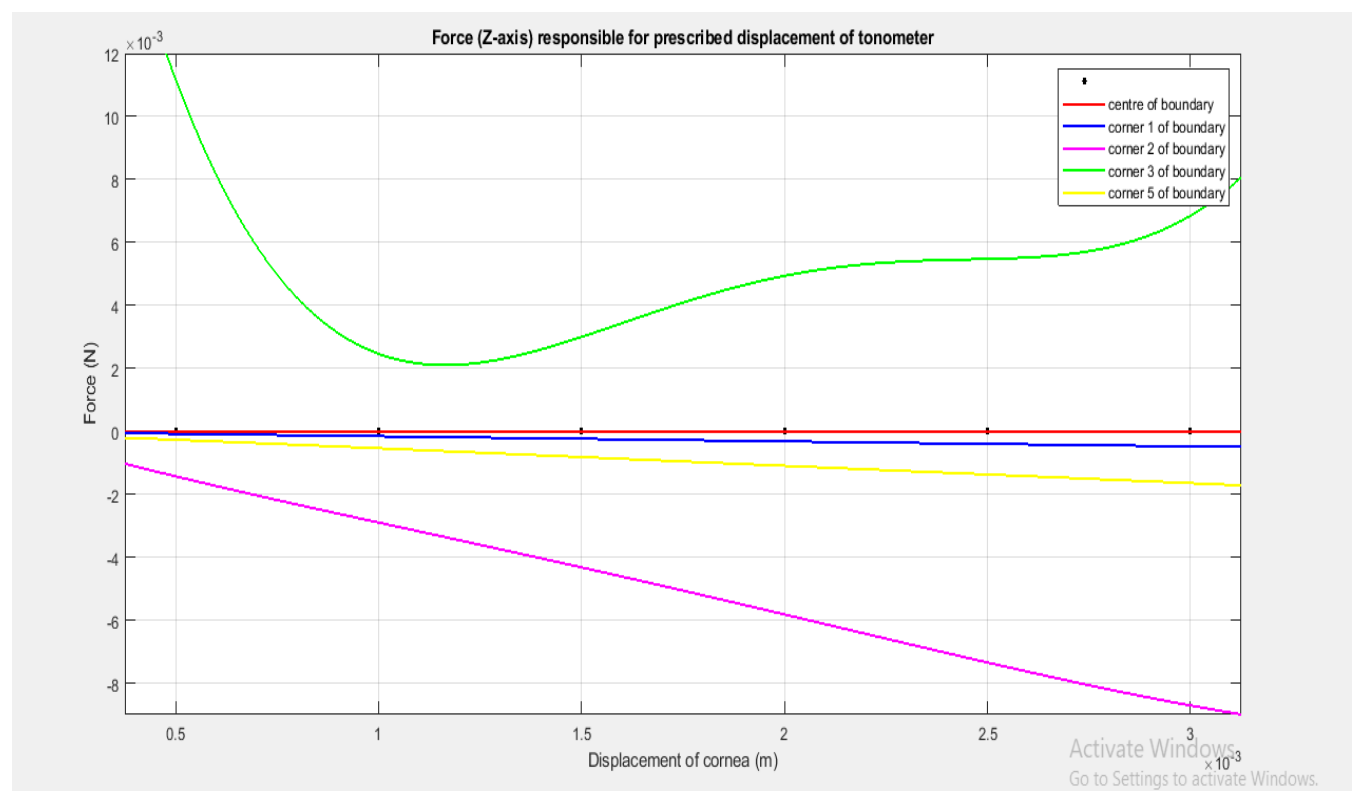

Figure 12. Force at the center and at 4 corner points of boundary layer along Z-axis

Table 4. Coefficients (p1, p2,p3, p4, p5) of (4) at the center and boundary points

\begin{tabular}{ccccccc}
\hline Boundary selection point & P1 & P2 & P3 & P4 & P5 & $R^{2}$ \\
\hline Centre & $-1.986 \mathrm{e}-09$ & 29.63 & -0.1556 & -0.003257 & $-3.333 \mathrm{e}-08$ & 1 \\
Corner 1 & $-7.333 \mathrm{e}+05$ & 5578 & -14.25 & -0.1472 & $-2.5 \mathrm{e}-06$ & 1 \\
Corner 2 & $1.008 \mathrm{e}+08$ & $-6.538 \mathrm{e}+05$ & 1317 & -4.106 & 0.0003422 & 1 \\
Corner 3 & $3.367 \mathrm{e}+09$ & $-2.731 \mathrm{e}+07$ & $7.94 \mathrm{e}+04$ & -95.07 & 0.04207 & 0.9919 \\
Corner 4 & $-5.333 \mathrm{e}+06$ & $4.044 \mathrm{e}+04$ & -106 & -0.4423 & $-2.7 \mathrm{e}-05$ & 1 \\
\hline
\end{tabular}

\section{CONCLUSION}

The human eye is modeled using FEM based simulation software for determining the force on cornea responsible for the predetermined deformation of the cornea during tonometry and gonioscopy test procedures. The deformation of the cornea during tonometry and gonioscopy is considered in the range of $0.5 \mathrm{~mm}$ to $3 \mathrm{~mm}$ along X-axis. The study is carried out at constant normal IOP of $22 \mathrm{mmHg}$. The thickness of the cornea for a normal eye is considered to be $0.5 \mathrm{~mm}$. The graphical results conclude the following: (a) during tonometry, while force is applied at the center point of cornea, the force of $1.6 \mathrm{~N}$ is experienced for $3 \mathrm{~mm}$ displacement of the cornea along the $\mathrm{X}$-axis. There is no force experienced by the tonometer along $\mathrm{Y}$ and $\mathrm{Z}$ direction; (b) with tonometer or goniolens of $1 \mathrm{~mm}$ diameter spread across the center of cornea, the maximum force of $0.03 \mathrm{~N}$ is experienced at the corners of tonometer or goniolens and negligible force at the centre along $\mathrm{X}$ direction for $3 \mathrm{~mm}$ displacement of the cornea. The amount of force experienced along $\mathrm{Y}$ and $\mathrm{Z}$ axes at all the points of the boundary layer for $3 \mathrm{~mm}$ deformation of the cornea is negligible.

The simulation is carried out to determine the amount of force that will be applied over the surface of cornea during tonometry and gonioscopy tests. The excess force on the cornea may either result in damaging the internal parts of the eye or may end up with erroneous results. The observations on the quantified force can be utilized during the design of new technology for the evaluation of intraocular pressure (IOP) measurement or can also be implemented as an additional constituent in any working tonometer. In the future, the goniolens can also be embedded with an additional feature of tracking the force on cornea. The simulation results may not always give exact results as predicted because of linear and isotropic material properties of the eye. The future scope is to validate the observations in the simulation work on the cadaver or goat eyes. The force or cornea for the goat or cadaver eye is quantified during the fixed amount of deformation of the cornea or the amount of deformation is measured during the fixed amount of force on cornea. 


\section{REFERENCES}

[1] Coleman, A. L., and Miglior, S., "Risk factors for glaucoma onset and progression," Survey of Ophthalmology, vol. 53, no. 6, pp. S3-S10, 2008.

[2] Singh, P., Tyagi, M., Kumar, Y., Kuldeep, K., and Sharma, P. D., “Gonioscopy: A review," Open Journal of Ophthalmology, vol. 3, no. 4, pp. 118-121, 2013.

[3] Moses, R. A., "The Goldmann applanation tonometer," American journal of ophthalmology, vol. 46, no. 6, pp. 865-869, 1958.

[4] Rich, D., U.S. Patent Application No. 10/495,097, 2005.

[5] Majzoub, U., "Volume changes in indentation tonometry," The British journal of ophthalmology, vol. 51, no. 6, pp. 365-378, 1967.

[6] Stocker, F. W., "On Changes in Intraocular Pressure After Application of the Tonometer*: In the Same Eye and in the Other Eye," American journal of ophthalmology, vol. 45, no. 2, pp. 192-196, 1958.

[7] Ishiguro, S., and Morino, T., U.S. Patent No. 5,501,217. Washington, DC: U.S. Patent and Trademark Office, 1996.

[8] Chen, H., et al., "Numerical investigation of topical drug transport in the anterior human eye," International Journal of Heat and Mass Transfer, vol. 85, pp. 356-366, 2015.

[9] Tiang, K. L., and Ooi, E. H., "Effects of aqueous humor hydrodynamics on human eye heat transfer under external heat sources," Medical engineering and physics, vol. 38, no. 8, pp. 776-784, 2016.

[10] Cvetkovic, M., et al., "A simple finite element model of heat transfer in the human eye," IEEE International Conference on Software in Telecommunications and Computer Networks, pp. 27-31, 2006.

[11] Ooi, E. H., et al., "Bioheat transfer in the human eye: A boundary element approach," Engineering Analysis with Boundary Elements, vol. 31, no. 6, pp. 494-500, 2007.

[12] Akkar, S., et al., "Modeling Heat Transfer in the Eye during Cataract Surgery," Cornell university library, 2009.

[13] Kotha, S., and Murtomäki, L., "Virtual pharmacokinetic model of human eye," Mathematical biosciences, vol. 253, pp. 11-18, 2014.

[14] Ooi, E. H., and Ng, E. Y. K., "Simulation of aqueous humor hydrodynamics in human eye heat transfer," Computers in biology and medicine, vol. 38, no. 2, pp. 252-262, 2008.

[15] Dua, S., et al., "Computational analysis of the human eye with applications," World Scientific, 2011.

[16] Özcura, F., et al., "Evaluation of Goldmann applanation tonometry, rebound tonometry and dynamic contour tonometry in keratoconus," Journal of Optometry, vol. 10, no. 2, pp. 117-122 2017.

[17] Nakakura, S., et al., "Evaluation of corneal displacement using high-speed photography at the early and late phases of noncontact tonometry," Investigative ophthalmology \& visual science, vol. 54, no. 4, pp. 2474-2482, 2013.

[18] Shin, J., et al., "The effect of corneal biomechanical properties on rebound tonometer in patients with normaltension glaucoma," American journal of ophthalmology, vol. 159, no. 1, pp. 144-154, 2015.

[19] Koprowski, R., et al., "Selected parameters of the corneal deformation in the Corvis tonometer," Biomedical engineering online, vol. 13, no. 55, 2014. doi: https://doi.org/10.1186/1475-925X-13-55

[20] Kempf, R., et al., "Understanding eye deformation in non-contact tonometry," International Conference of the IEEE Engineering in Medicine and Biology Society, pp. 5428-5431, 2006.

[21] Moses, R. A., and Grodzki, W. J., "Theory and calibration of the Schiøtz tonometer: II. The resistance of the cornea to deformation," Investigative Ophthalmology \& Visual Science, vol. 10, no. 7, pp. 539-543, 1971.

[22] Ooi, E. H., and Ang, W. T., "A boundary element model of the human eye undergoing laser thermokeratoplasty," Computers in Biology and Medicine, vol. 38, no. 6, pp. 727-737, 2008.

[23] Kempf, R., et al., "Understanding eye deformation in non-contact tonometry," In 2006 International Conference of the IEEE Engineering in Medicine and Biology Society, IEEE, pp. 5428-5431, 2006.

[24] Y. Kurita et al., "Contact-Based Stiffness Sensing of Human Eye," in IEEE Transactions on Biomedical Engineering, vol. 55, no. 2, pp. 739-745, Feb. 2008.

[25] Friedman, M. D., Muller, D., and Paranjape, A., U.S. Patent No. 9,498,114. Washington, DC: U.S. Patent and Trademark Office, 2016. 